\title{
Two new species of Amynthas (Clitellata: Megascolecidae) from lettuce fields of Mt. Taebaek, Korea
}

\author{
Yong HONG \\ Department of Agricultural Biology, College of Agriculture \& Life Sciences, \\ Chonbuk National University, Jeonju 561-756, Korea
}

\begin{abstract}
Two new species of Amynthas (Clitellata: Megascolecidae) from lettuce fields of Mt. Taebaek, Korea. - Two new species of earthworms were found in a survey of lettuce cultivation fields, created by slash and burn. Amynthas hasamensis sp. n. and Amynthas samgaki sp. n. have simple intestinal caeca and no genital markings. Amynthas hasamensis sp. n. keys to the hawayanus group in Sims \& Easton (1972) with three pairs of spermathecae in VI, VII, and VIII. Its male field areas in XVIII are complex with paired oval male discs bearing two transverse ridges with seminal grooves between the ridges. Amynthas samgaki sp. n. keys to the morrisi group with two pairs of spermathecae in VI and VII, and has a male field with large egg-shaped raised pads with transverse seminal grooves.
\end{abstract}

Keywords: Earthworms - Amynthas - Megascolecidae - Clitellata - Korea lettuce fields - taxonomy.

\section{INTRODUCTION}

Korean earthworm communities in forests and agricultural ecosystems are dominated by species of the genus Amynthas (Megascolecidae). Many new Korean species have been described recently, further expanding the genus Amynthas (Hong \& James, 2001a, b; Hong \& Lee, 2001; Hong et al., 2001; Hong \& Kim, 2002a, b; Hong, 2007; Hong \& James, 2009). The shape of the male pore region, especially the male discs, is useful for taxonomy of Korean Amynthas and has been used throughout the history of this taxon. Species with male discs were reported in Hong \& James (2001a) and Hong (2007). In this study, I describe two species with male discs: Amynthas hasamensis sp. n. and Amynthas samgaki sp. n. Exotic Drawida species (Moniligastridae) were also found in the area, so I conclude that endemic and exotic species are living together. An earlier report of the earthworm fauna from Mt. Taebaek found only one species, Amynthas taebaekensis (Hong \& James, 2001a).

Mt. Taebaek is located to the north of Seoul in Gangwon Provincial Park, which has natural forests. Among its big mountains are Janggun peak (1,567 m), Munsoo peak $(1,517 \mathrm{~m})$, and Busoe peak $(1,546 \mathrm{~m})$. Specimens were collected by digging and hand sorting in June 2006 and July 2007 in slash-and-burn fields used for lettuce cultivation. Taxonomy in this paper follows Sims \& Easton (1972), and Easton (1979). In the location data the terms "dong" and "ri" refer to successively smaller Korean political divisions and do not have direct English equivalents. The "ri" division is 
preceded by a number if there is more than one ri of a particular name. In this paper, "Hasam 2-ri" indicates the second ri named Hasam. Illustrations are of anatomical views containing important features, prepared with a camera lucida. Descriptions are based on the external examination and dorsal dissection under the stereomicroscope. Holotype and some paratypes are deposited in the National Institute of Biologocal Resources, Korea (NIBR), and other paratypes are deposited in the Museum of Natural History of Geneva (MHNG).

\section{DESCRIPTION}

Family Megascolecidae Rosa, 1891

Genus Amynthas Kinberg, 1867

Amynthas hasamensis sp. $\mathrm{n}$.

Figs 1A-B

Material: Holotype; clitellate (NIBRIV0000224640); Korea, Gangwon province, Taebaek municipality, Samsu-dong, Hasam 2-ri, Mt. Taebaek ( $\left.37^{\circ} 14.074^{\prime} \mathrm{N}, 128^{\circ} 59.151^{\prime} \mathrm{E}\right)$, 600-800 m, fields cleared for cultivation by burning, soil and litter layers, 29 June 2006, Y. Hong coll. - Paratypes: 1 clitellate (NIBRIV0000224641), 1 clitellate (MHNG INVE 75339); same data as for holotype. - Nontype material; 2 clitellate specimens, Taebaek municipality, Samsudong, Hasam 2-ri, Mt. Taebaek ( $\left.37^{\circ} 14.074^{\prime} \mathrm{N}, 128^{\circ} 59.151^{\prime} \mathrm{E}\right), 600-800 \mathrm{~m}$, soil and litter layers, 4 July 2007, Y. Hong coll.

ETYMOLOGY: The species is named for Hasam 2-ri, its type locality.

DiaGnOsis: Three pairs of spermathecal pores in 5/6-7/8; male discs each with two transverse low ridges, short diagonal seminal groove between ridges, no other genital markings.

DESCRIPTION: Dimensions $80-130 \mathrm{~mm}$ by $5.5-6.5 \mathrm{~mm}$ at segment $\mathrm{X}, 6.0-6.5 \mathrm{~mm}$ at segment XXX, 6.0-8.0 mm at clitellum; body cylindrical, segments 59-109. Setae regularly distributed around segmental equators numbering 31-35 at VII, 63-66 at XX, 0 between male pores, setal formula AA:AB:ZZ:YZ= 2:2:4:3 at XIII. Female pore single in XIV, within $0.8-1.0 \mathrm{~mm}$ circular porophore. Prostomium epilobic with tongue open, clitellum coffee color, formalin preservation. First dorsal pores at 12/13, one individual 11/12. Clitellum annular XIV-XVI; setae invisible externally.

Male field composed of paired male discs, each approximately ovate with longer axis transverse; each disc with two narrow transverse ridges, one equatorial, centered to slightly lateral of center on disc; one on posterior margin of disc, almost as long as long axis of disc. Surface between ridges and within outer margins of discs generally depressed; midventral gap between discs even more depressed. Seminal grooves short, from just posterior to medial end of equatorial ridge, diagonally posterior, lateral in a short arc convex anterior-laterally across depressed area between ridges to meet posterior ridge. Male pores at medial ends of seminal grooves, close to equatorial ridges, 0.15 circumference apart. Spermathecal pores in 5/6-7/8, 0.16 circumference apart, each with small tongue-like appendage within furrow; thickened lips immediately adjacent to each pore; ventral surface of VI-VIII thickened, slightly rugose, faintly pigmented.

Septa 5/6-7/8 thick, 8/9/10 absent, 10/11-13/14 thinly muscular. Gizzard large in VIII-X. Intestine begins in XV; lymph glands not found. Typhlosole medium sized 


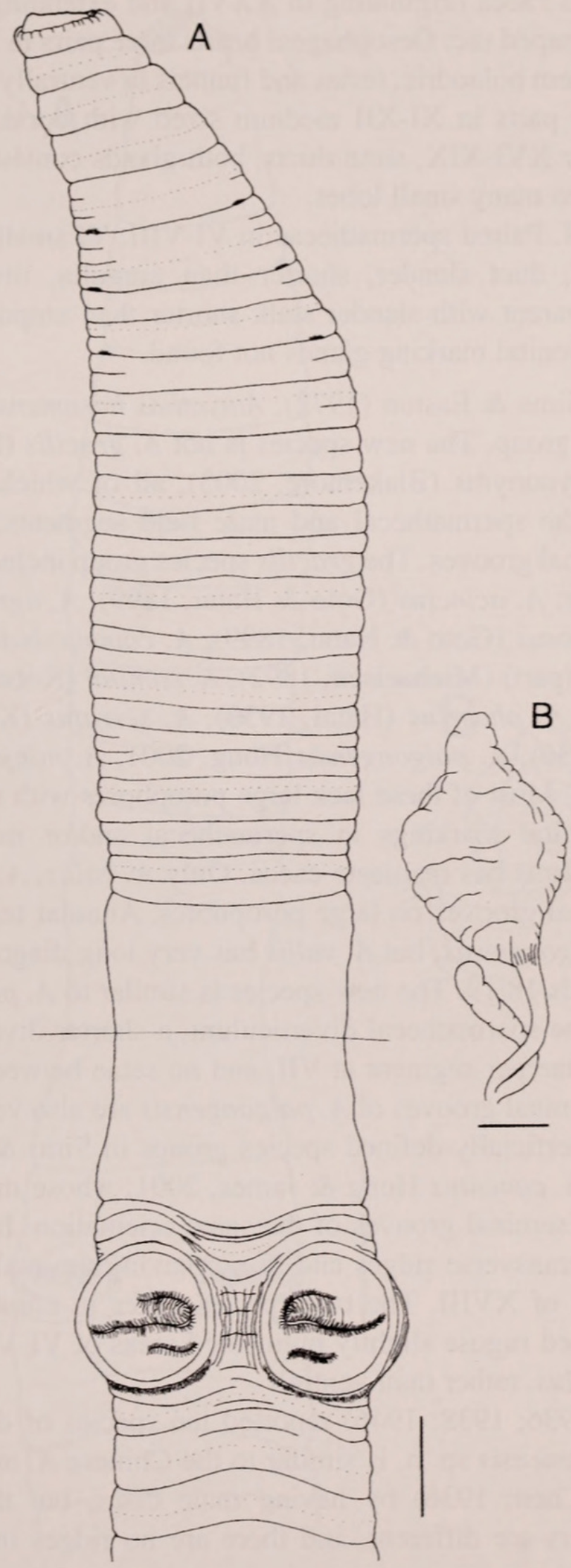

FIG. 1

Amynthas hasamensis sp. $\mathrm{n}$. (A) Ventral view. (B) Spermathecae and diverticulum. Scale bars $=$ $2.5 \mathrm{~mm}(\mathrm{~A}), 2 \mathrm{~mm}(\mathrm{~B})$. 
from XXVI. Intestinal caeca originating in XXVII and extending anteriorly about to XXII, simple finger-shaped sac. Oesophageal hearts three pairs in XI-XIII; X, only one side. Male sexual system holandric, testes and funnels in ventrally joined sacs in X-XI. Seminal vesicles two pairs in XI-XII medium sized with dorsal lobes. Prostates in XVIII, extending over XVI-XIX, stout ducts, both glands consist of two main lobes, each divided again into many small lobes.

Ovaries in XIII. Paired spermathecae in VI-VIII, VI smallest; ampulla surface wrinkled egg-shaped, duct slender, shorter than ampulla, diverticulum chamber clavate, almost transparent with slender stalk shorter than ampulla, no nephridia on spermathecal ducts. Genital marking glands not found.

REMARKS: In Sims \& Easton (1972), Amynthas hasamensis sp. n. keys to the hawayanus (gracilis) group. The new species is not A. gracilis (Kinberg 1867) or its possible and actual synonyms (Blakemore, 2003), all of which have several small genital markings in the spermathecal and male field segments, and lack the large porophores with seminal grooves. The gracilis species group includes 13 species found in Korea and/or Japan: A. acinctus (Goto \& Hatai, 1899), A. agrestis (part) (Goto \& Hatai, 1898), A. carnosus (Goto \& Hatai, 1899), A. communissimus (Goto \& Hatai, 1899), A. hilgendorfi (part) (Michaelsen, 1892), A. kamitai (Kobayashi, 1934), A. pa pulosus (Rosa,1896), A. phaselus (Hatai, 1930), A. serratus (Kobayashi, 1936), A. vallis (Kobayashi, 1936), A. palgongensis Hong, 2001, A. minjae Hong, 2001, and A. jamesi Hong, 2007. Most of these lack large porophores with seminal grooves and most have small genital markings in spermathecal and/or male field segments. Amynthas communissimus has manicate caeca. Only A. vallis, A. jamesi, and A. palgongensis have seminal grooves on large porophores. Annular testes sacs are present in $A$. vallis and $A$. palgongensis, but $A$. vallis has very long diagonal seminal grooves extending back towards $18 / 19$. The new species is similar to A.palgongensis, but has a different shape of the spermathecal diverticulum, a shorter diverticulum relative to the ampulla, fewer setae per segment at VII, and no setae between male pores ( 0 vs. 1-4). The T-shaped seminal grooves of A. palgongensis are also very distinct. Looking beyond the rather superficially-defined species groups in Sims \& Easton (1972), the species is similar to A. cuneatus Hong \& James, 2001, whose male field consists of male discs with short seminal grooves of the same orientation. It differs from $\mathrm{A}$. $\mathrm{cu}$ neatus in having the transverse ridges and in not having the male discs mounted on large alate extensions of XVIII. The two species differ in number of spermathecal pores, and the thickened rugose slightly pigmented areas of VI-VIII (genital patches) are lateral in A. cuneatus, rather than ventral.

Chen $(1933 ; 1936 ; 1938 ; 1946)$ reported ten species of the gracilis group in China. Amynthas hasamensis sp. n. is similar to the Chinese A. muticus (Chen, 1938) and A. magnificus (Chen, 1936) by having male discs, but the disc shapes and spermathecal characters are different, and there are no ridges in the discs of either Chinese species.

MATERIAL: Holotype; clitellate (NIBRIV0000224642); Korea, Gangwon province, Taebaek municipality, Samsu-dong, Hasam 2-ri, Mt. Taebaek (37 $\left.14.074^{\prime} \mathrm{N}, 128^{\circ} 59.151^{\prime} \mathrm{E}\right)$, 


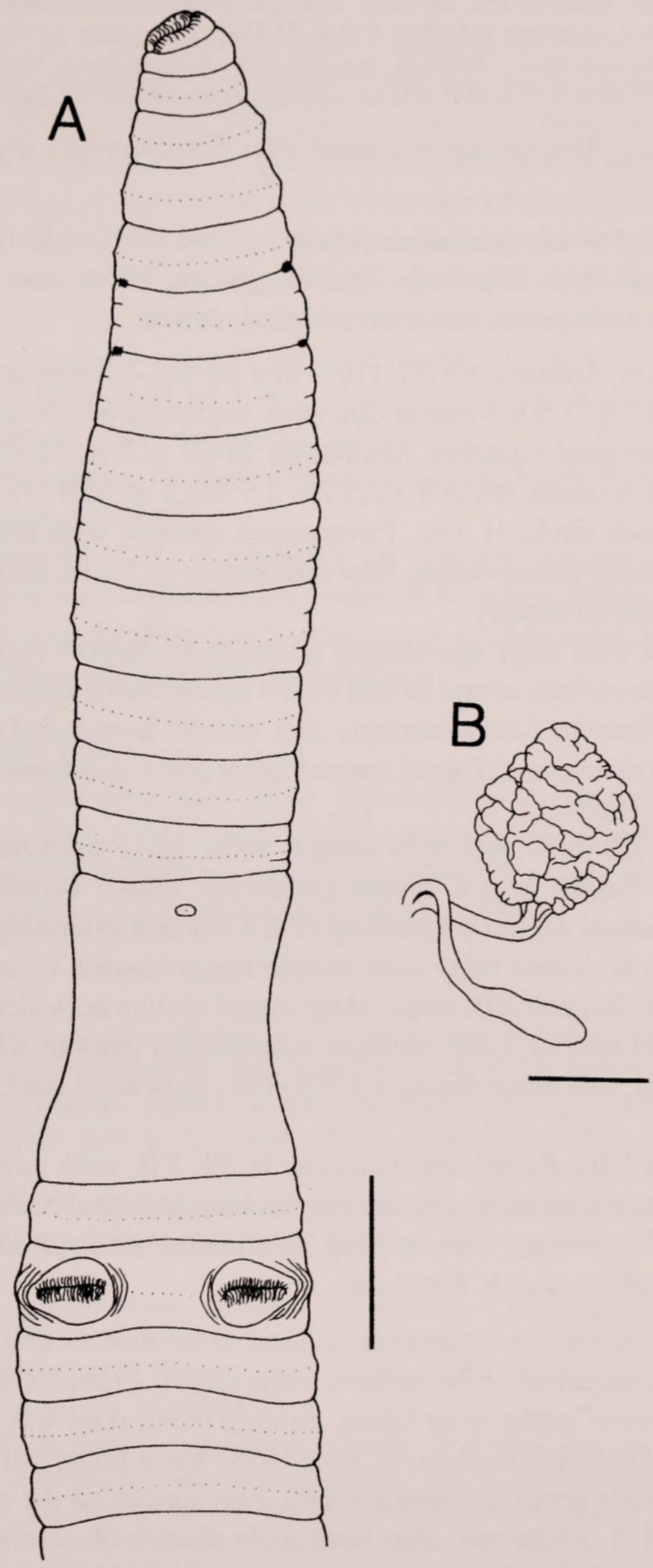

FIG. 2

Amynthas samgaki sp. n. (A) Ventral view. (B) Spermathecae and diverticulum. Scale bars = $4 \mathrm{~mm}$ (A), $2 \mathrm{~mm}$ (B). 
600-800 m, soil and litter layers, 29 June 2006, Y. Hong coll. - Paratypes; 1 clitellate (NIBRIV0000224643), 1 clitellate (MHNG INVE 75338); same data as for holotype. - Nontype material; 1 clitellate specimen, Taebaek municipality, Samsu-dong, Hasam 2-ri, Mt. Taebaek ( $\left.37^{\circ} 14.074^{\prime} \mathrm{N}, 128^{\circ} 59.151^{\prime} \mathrm{E}\right), 600-800 \mathrm{~m}$, soil and litter layers, 4 July 2007 , Y. Hong coll.

Etymology: The species is named after Sim Samgak, whose farm is its type locality.

Diagnosis: Paired spermathecal pores in 5/6, 6/7; male field with large eggshaped raised pads with transverse seminal groove across pad center, $2.5-3.0 \mathrm{~mm}$ distance between male pores, testes sacs ventral, paired.

DESCRIPTION: Dimensions 77-110.5 $\mathrm{mm}$ by 4.5-4.7 $\mathrm{mm}$ at segment $\mathrm{X}, 6.0-6.5$ $\mathrm{mm}$ at segment XXX, 5.5-6.5 mm at clitellum; segments 64-79. Setae regularly distributed around segmental equators, numbering 24-32 at VII, 52-63 at XX, 7 between male pores, setal formula $\mathrm{AA}: \mathrm{AB}: \mathrm{ZZ}: \mathrm{YZ}=2.5: 2: 2: 2$ at XIII. Female pore single in $\mathrm{XIV}$, on 0.6-0.9 mm circle or oval. Prostomium epilobic with tongue open, clitellum coffee color, formalin preservation. First dorsal pore at 12/13. Clitellum annular XIVXVI; setae invisible externally.

Male field with large egg-shaped raised pads, narrow end lateral, with transverse seminal groove from center of pad to just inside lateral edge, male pore at medial end of groove. Three to four concentric arcs around lateral end of pad, 2.5-3.0 mm distance between male pores. Paired spermathecal pores in $5 / 6$ and $6 / 7$, lateral, minute. Genital markings lacking.

Septa 5/6-7/8 thick, 8/9, 9/10 absent, 10/11-12/13 thickened. Gizzard large in VIII-X. Intestine begins in XV, lymph glands not found. Typhlosole medium sized from XXVI. Intestinal cecum originating in XXVII and extending anteriorly about to XXIV, bent down to ventral body wall, simple finger-shaped. Oesophageal hearts four pairs in X-XIII; X, XII and XIII large. Male sexual system holandric, testes and funnels in ventrally paired sacs in X-XI. Seminal vesicles two pairs in XI-XII medium sized. Prostates in XVIII, extending through XVII-XIX, long stout duct, glands divided into small lobes.

Ovaries in XIII. Paired spermathecae in VI, VII; each ampulla roughly ovate, flattened, wrinkled on surfaces, slender duct as long as ampulla; diverticulum chamber club-shaped, white; slender stalk as long as ampulla, no nephridia on spermathecae ducts. Genital marking glands not found.

REMARKS: Amynthas samgaki sp. n. keys to the morrisi group in Sims \& Easton (1972), which is composed of 30 species. Chen $(1933 ; 1936 ; 1938 ; 1946)$ recorded 12 species of the morrisi group from China. Among them, none has male pores on discs or similar pads. Amynthas oculatus (Chen, 1938) has a large pair of postsetal genital papillae but the male pores lie outside these. Two species of the canaliculatus-group, A. jangbogoi and A. jindoensis, also have male discs with seminal grooves, and the pairs of spermathecal pores are intrasegmental on VI and VII, rather than in 5/6/7. In A. jangbogoi the disc is bowling-pin shaped. The spermathecal diverticulum stalk of the latter has numerous tight kinks, as opposed to straight. I include this comparison because the canaliculatus-group could include morrisi-group species with independently evolved intrasegmental spermathecal pores. 
The following Korean morrisi-group species are known: A. fibulus fibulus (Kobayashi, 1936), A. fibulus ranunculus (Kobayashi, 1936), A. kobayashii (Kobayashi, 1938), A. koreanus (Kobayashi, 1938), A. geojeinsulae (Song \& Paik, 1970), A. draconis Hong \& James, 2001, A. naejangensis Hong \& James, 2001, A. assimilis Hong \& Kim 2002, and A. gyeryongensis Hong \& Kim, 2002. Amynthas assimilis and A. naejangensis have male discs with seminal grooves. In A. assimilis the disc and groove are oriented diagonally. Amynthas naejangensis is larger and has more segments (116-153 $\mathrm{mm}$ vs 77-110 mm, 96-117 vs 64-79 respectively) but is very similar in most respects. The following details of A. naejangensis are to be contrasted to the description of Amynthas samgaki sp. n.: testes sacs dorsally united in X and XI, and enclosing seminal vesicles of XI, lymph glands present, male discs encroaching on adjacent segments, dorsal setal gap present, as indicated in the setal formula $\mathrm{YZ}: \mathrm{ZZ}=$ 2.5:4. Clearly the two species are quite similar and could be regionally-differentiated descendants of a common ancestor.

Amynthas samgaki sp. $\mathrm{n}$. is probably an endemic species, but more distributional and ecological data are needed to establish its relationships to soils and land use patterns. Further investigations are needed on its phylogenetic relationships with other Amynthas species, particularly A. naejangensis.

\section{ACKNOWLEDGEMENTS}

The author would like to express appreciation to Dr Samuel W. James, University of Kansas, USA, who kindly shared valuable bionomical information and reviewed the taxonomic descriptions in the manuscript. This work was supported by a grant from Rural Development Administration, Korea (2006).

\section{REFERENCES}

BlaKemoRe, R. J. 2003. Japanese earthworms (Annelida: Oligochaeta): a review and checklist of species. Organisms, Diversity and Evolution 3. Electronic Supplement 11: 1-43.

CHEN, Y. 1933. A preliminary survey of the earthworms of the Lower Yangtze valley. Contributions of the Biology Laboratory Sciences Society of China (Zoology), Nanking 9: 178-296.

CHEN, Y. 1936. On the terrestrial Oligochaeta from Szechwan II with the notes on Gates' types. Contributions of the Biology Laboratory Sciences Society of China (Zoology), Nanking 11: 269-306.

CHEn, Y. 1938. Oligochaeta from Hainan, Kwangtung. Contributions from Biological Laboratory of Science Society of China (Zoological series) 12: 375-427.

CHEN, Y. 1946. On the terrestrial Oligochaeta from Szechwan III. Journal of the West China Border Research Society 16: 83-141.

Easton, E. G. 1979. Acaecate earthworms of the Pheretima group (Megascolecidae: Oligochaeta): Archipheretima, Metapheretima, Planapheretima, Pleionogaster and Polypheretima. Bulletin of the British Museum of Natural History (Zoology) 35(1): 1-126.

Hong, Y. 2007. Some new earthworms of the genus Amynthas (Oligochaeta: Megascolecidae) with male discs from Bogildo Island, Korea. Revue suisse de Zoologie 114: 721-728.

Hong, Y. \& JAmES, S. W. 2001a. New species of Korean Amynthas Kinberg, 1867 (Oligochaeta, Megascolecidae) with two pairs of spermathecae. Revue suisse de Zoologie 108: 65-93.

Hong, Y. \& JAmES, S. W. 2001b. Five new earthworms of the genus Amynthas Kinberg (Megascolecidae) with four pairs of spermathecae. Zoological Studies 40: 269-275. 
Hong, Y. \& JAmES, S. W. 2009. Some new Korean megascolecoid earthworms (Oligochaeta). Journal of Natural History 43: 1229-1256.

HonG, Y. \& KIM, T. H. 2002a. Three new earthworms of the genus Amynthas (Megascolecidae) from Mt. Gyeryong, Korea. Revue suisse de Zoologie 109: 483-489.

Hong, Y. \& KIM, T. H. 2002b. Four new earthworms of the genus Amynthas (Oligochaeta: Megascolecidae) from Korea. Korean Journal of Biological Science 6: 195-199.

HonG, Y. \& LeE, W. K. 2001. Description of three new Korean earthworms of the genus Amynthas Kinberg, 1867 (Oligochaeta, Megascolecidae) with multiple genital markings. Revue suisse de Zoologie 108: 283-290.

Hong, Y., LeE, W. K. \& Kim, T. H. 2001. Four new species of the genus Amynthas Kinberg (Oligochaeta: Megascolecidae) from Korea. Zoological Studies 40: 263-268.

Sims, R. W. \& EASTON, E. G. 1972. A numerical revision of the earthworm genus Pheretima auct. (Megascolecidae: Oligochaeta) with the recognition of new genera and an appendix on the earthworms collected by the Royal Society North Borneo Expedition. The Biological Journal of the Linnean Society, London 4: 169-268. 


\section{$2 \mathrm{BHL}$ Biodiversity Heritage Library}

Hong, Yong. 2011. "Two new species of Amynthas (Clitellata: Megascolecidae) from lettuce fields of Mt. Taebaek, Korea." Revue suisse de zoologie 118, 223-230. https://doi.org/10.5962/bhl.part.117806.

View This Item Online: https://www.biodiversitylibrary.org/item/148548

DOI: https://doi.org/10.5962/bhl.part.117806

Permalink: https://www.biodiversitylibrary.org/partpdf/117806

\section{Holding Institution}

American Museum of Natural History Library

\section{Sponsored by}

BHL-SIL-FEDLINK

\section{Copyright \& Reuse}

Copyright Status: In copyright. Digitized with the permission of the rights holder.

Rights Holder: Muséum d'histoire naturelle - Ville de Genève

This document was created from content at the Biodiversity Heritage Library, the world's largest open access digital library for biodiversity literature and archives. Visit BHL at https://www.biodiversitylibrary.org. 